\title{
Surgical Approach to Recurrent Cholangiocarcinoma
}

\author{
Hauke Lang ${ }^{a}$ \\ a Department of General, Visceral and Transplant Surgery, University Medical Center of the Johannes Gutenberg \\ University Mainz, Mainz, Germany
}

\section{Keywords}

Cholangiocarcinoma $\cdot$ Tumor recurrence $\cdot$ Repeated resection - Treatment of recurrence $\cdot$ Survival

\begin{abstract}
Background: For recurrent cholangiocarcinoma, systemic chemotherapy is the standard of care. Repeated resection is a potential curative treatment, but data are scarce and outcomes are not well defined so far. Summary: In the last decade there has been an increasing number of reports suggesting a survival benefit and even cure after repeated surgery. This is particularly true for intrahepatic cholangiocarcinoma, where repeated resections offer similar or even better results than the first resection. In selected cases even a third liver resection is possible. In contrast, in perihilar and distal cholangiocarcinoma, repeated resection is only rarely possible. Although the improved outcome might be attributed to a careful patient selection and a favorable tumor biology, repeated surgical treatment should be attempted whenever possible. This necessitates a structured follow-up after resection. Key Message: Surgical resection offers the chance for prolonged survival and even for cure in recurrent cholangiocarcinoma. Hence, it is of the utmost importance to better understand the routes of metastases and to define factors qualifying for resection. Whether different patterns of recurrence reflect biological heterogeneity requires further investigation.

(c) 2020 S. Karger AG, Basel
\end{abstract}

\section{Introduction}

Cholangiocarcinoma is a rare malignancy arising from the epithelium of the intra- or extrahepatic biliary tract. It accounts for about $1 \%$ of gastrointestinal carcinomas. Tumors in the biliary tree proximal to the left or right hepatic duct are classified as intrahepatic (iCCA), and those located more distally till the junction with the cystic duct are classified as perihilar cholangiocarcinoma (pCCA). Cancers of the biliary tract distal from the cystic duct are named distal cholangiocarcinoma (dCCA). Nearly half of bile duct tumors refer to pCCA, followed by iCCA. Distal bile duct cancers are less frequent, accounting for about $10-15 \%$ of cases [1-3].

Radical surgical resection is the treatment of choice for all types of cholangiocarcinoma. However, despite continuous advances in surgical techniques as well as newly available adjuvant therapies, more than half of the patients develop tumor recurrence which is associated with a poor outcome [4-7].

Traditionally, patients with recurrent cholangiocarcinoma have been treated with a palliative intention, most often with best supportive care only. Currently, chemotherapy, usually triple regimens based on gemcitabine/ cisplatin/nab paclitaxel or fluorouracil, leucovorin, and irinotecan plus oxaliplatin, if possible in combination with the antiangiogenic inhibitor bevacizumab, is the mainstay of treatment in advanced and also recurrent cholangiocarcinoma $[1,2,8]$. With the development of next-generation sequencing (NGS), molecular targeted 
Table 1. Review of the literature on repeated surgery for cholangiocarcinoma

\begin{tabular}{|c|c|c|c|c|c|c|c|}
\hline \multirow[t]{2}{*}{ Reference } & \multirow[t]{2}{*}{ Year } & \multirow[t]{2}{*}{ Patients, $n$} & \multirow[t]{2}{*}{ Primary disease } & \multicolumn{4}{|l|}{ Survival } \\
\hline & & & & median, months & 1-year, \% & 3 -year, \% & 5 -year, \% \\
\hline Yamamoto et al. [14] & 2001 & 4 & iCCA & 10 & & 29 & 29 \\
\hline Sotiropoulos et al. [15] & 2005 & 3 & $\mathrm{iCCa}$ & & 100 & 66.6 & 33.3 \\
\hline Ohtsuka et al. [16] & 2009 & 9 & iCCA & 22 & & 51.4 & 51.4 \\
\hline Ercolani et al. [17] & 2010 & 6 & iCCA & 66.6 & & 60 & 0 \\
\hline Kamphues et al. [12] & 2010 & 9 & iCCA & 51 & & 52 & 29 \\
\hline Song et al. [6] & 2011 & 27 & 5 iCCa, 9 pCCA, 13 dCCA & 18.9 & & & \\
\hline Saiura et al. [18] & 2011 & 5 & iCCA & & & 80 & 60 \\
\hline Sulpice et al. [19] & 2012 & 4 & $\mathrm{iCCa}$ & & & 100 & 75 \\
\hline Hyder et l. [20] & 2013 & 33 & iCCA & 25.8 & & & \\
\hline Zhang et al. [13] & 2013 & $32 / 77 \#$ & iCCA & $20.3 / 21.3^{\#}$ & $83.8 / 69.8^{\mathrm{c}}$ & $17.1 / 20.5^{\mathrm{c}}$ & na \\
\hline Takahashi et al. [7] & 2015 & 54 & 7 iCCA, 34 pCCA, 13 dCCA & & 70 & 38 & 18 \\
\hline Noji et al. [21] & 2015 & 18 & $\mathrm{pCCA} / \mathrm{dCCA}$ & & & 25 & 12 \\
\hline Spolverato et al. [22] & 2016 & 41 & iCCA & 26.1 & na & na & na \\
\hline Si et al. [23] & 2017 & 72 & $\mathrm{iCCa}$ & - & 97.2 & 67 & 41.9 \\
\hline Miyazaki et al. [24] & 2017 & 13 & 9 iCCA, 3 pCCA, 1 dCCA & 28.5 & & 38 & 19 \\
\hline Bartsch et al. [25] & 2019 & 18 & iCCA & 39.4 & 81 & 55 & 37 \\
\hline Hu et al. [27] & 2019 & $132\left(88^{*} / 44^{* *}\right)$ & iCCA & $48.7^{\mathrm{a}} / 7.6^{\mathrm{b}}$ & & & $11.4^{\mathrm{a}} / 0^{\mathrm{b}}$ \\
\hline Yoh et al. [28] & 2019 & 15 & iCCA & 91.6 & & 86.7 & \\
\hline Bartsch et al. [29] & 2020 & 113 & iCCA & 36.8 & & 51 & 34 \\
\hline
\end{tabular}

na, not available. ${ }^{\mathrm{a}} \mathrm{R} 0 .{ }^{\mathrm{b}} \mathrm{R} 1$ or R2. ${ }^{\mathrm{c}}$ Treated by radiofreqency ablation.

therapies have become increasingly available. For iCCA, the first promising results have been reported after treatment with fibroblast growth factor receptor and isocitrate dehydrogenase inhibitors. Other targeted therapies such as anti-human epidermal growth factor receptor-2 inhibitors or polyadenosine diphosphate ribose polymerase inhibitors may play a role in the future [8]. Despite ongoing improvements and some remarkable advances - i.e., a median survival of up to 21 months in a subgroup of patients treated with a fibroblast growth factor receptor inhibitor - results are still poor and survival is hardly longer than 12 months $[1,2,8,9]$.

In recent years, radiological interventions have gained increasing importance in the treatment of recurrent cholangiocarcinoma confined to the liver [1,10-13]. While the main aim of transarterial chemoembolization (TACE) and selective internal radiotherapy (SIRT) is local control of tumors rather than cure, ablative treatment modalities may be potentially curative at least in small tumors. Occasionally, external radiation may be an option in highly selected cases with circumscribed tumors [1, 10-13].

In the last decade there has been an increasing number of reports suggesting a survival benefit and even cure after repeated surgery, mostly for iCCA $[6,7,14-29]$. Hence, it is of utmost importance to better understand patterns of recurrence and to identify those factors qualifying for repeated resection. The aims of this review are to give an update on surgical therapy of recurrent cholangiocarcinoma and to point out future perspectives.

\section{Perihilar Cholangiocarcinoma}

After potentially curative resection of pCCA, tumors recur in more than half of the cases, most often within the first 2 postoperative years. Even so, only little information is available regarding the patterns of metastatic spread [6, $7,21,24,30-32]$. In a specific analysis summarizing data from 2 high-volume centers (Memorial Sloan Kettering Cancer Center, NY, USA; Academic Medical Center, Amsterdam, The Netherlands) local tumor relapse was found in the hilum, in the bilioenteric anastomosis, or at the resection margin in $26 \%$ of cases. The most common sites of distant metastases were: lymph nodes, the liver away from the resection margin, the peritoneum, and the lungs with almost equal frequencies. It is noteworthy that $28 \%$ of patients developed tumor recurrence more than 5 years after the primary resection [31].

Komaya et al. [32] from Nagoya, Japan, further stratified patterns of recurrence according to the radicality of the initial resection. They reported a recurrence rate of $58.2 \%$ after $\mathrm{R} 0$ resections and of $80.6 \%$ after R1 resections, respectively. After R0 resections, distant metastases were more often the initial site of relapse, while after R1 resections there was a 3 -fold higher incidence of locoregional recurrence. Furthermore, the series from Nagoya observed recurrent pCCA after more than 5 years in $9 \%$ of the patients [32].

Since recurrent pCCA is usually either locoregional with involvement of hilar structures or multifocal and 
distant, a surgical approach is difficult. Accordingly, the goals of treatment are mainly control or at least attenuation of symptoms, i.e., relief of jaundice caused by a malignant biliary obstruction, therapy of cholangitis, local tumor control, and prevention of tumor progression. This is the domain of interventional radiology orendoscopy and of systemic treatment with chemotherapy and antibiotics.

Only few data about surgical treatment are available [6, $7,21,24]$ (Table 1). In the series from Nagoya, repeated resection was possible in $9.9 \%$ of the patients. All but 1 surgery were performed for distant metastases, with nearly half of them in the thoracic/abdominal wall [7]. Song et al. [6] reported 9 reresections, with 5 of them for seedings in the wall of the abdomen or chest. Specific survival data are not given (results are mixed with dCCA) but in both groups the survival after surgery was better than after palliative treatment (Table 1 ).

These data suggest that there is only a small place for surgery in recurrent pCCA. In local relapse a surgical approach is hardly possible. On contrast, in selected cases with distant, preferably solitary metastases, surgery might be reasonable. A special situation refers to tract seedings in the abdominal and chest wall. These metastases are often troublesome because of eruption and pain. Here, resection may be indicated to treat or to prevent symptoms and increase the quality of life rather than to achieve cure.

\section{Intrahepatic Cholangiocarcinoma}

Intrahepatic cholangiocarcinoma is the second most common bile duct carcinoma. Since jaundice is a rare and mostly late symptom, tumors usually remain undetected for a long period and the diagnosis is made in advanced stages. In primary iCCA mainly extended liver resections are required for complete tumor removal, often in combination with resections of vascular or hilar structures [4]. Hence, it is not surprising that most patients develop tumor recurrence also after R0-resection. However, in contrast to pCCA and dCCA patterns of recurrence have been studied by various groups, and the reported results are more or less concordant [22, 25-27]. Approximately, $50-70 \%$ of iCCA recur within a median time of 12 months after potentially curative resection [22, 25, 27]. The liver is the most common site of recurrence. In nearly half of the cases there is isolated hepatic recurrence with equal frequencies at the resection margin and at different sites in the liver. Sole extrahepatic metastases are found in about $10-25 \%$ of cases. The location of recurrent tumors seems to differ according to the time of appearance. Intrahepatic margin recurrence and diffuse intra- and extrahepatic relapse are more often seen in the early postoperative period. Similar to pCCA, in a substantial num- ber of cases there is late, mainly intrahepatic recurrence at sites different from the initial one.

In general, the prognosis of patients with recurrent iCCA is poor, and despite advances in almost all fields of cancer therapy the median survival is usually no longer than 12 months $[8,22,27]$. For many years, palliative chemotherapy or best supportive care has been the standard of care. The guidelines of the European Association for the Study of the Liver (EASL) from 2014 are the first to concede the possibility of either ablation or reresection [1].

Nevertheless, in recent years there have been several reports on surgery for recurrent iCCA [23, 25-29]. One of the largest series so far includes multicentric data from 17 German HPB centers with 113 resections, mainly minor hepatectomies or segmentectomies, resulting in a 3and 5-year disease-free survival rates of 36 and $28 \%$, respectively [29]. Similar results have been reported by several other authors [22, 23, 25-28] (Table 1). These survival rates are even slightly better than those after primary resection, thus justifying repeated surgery. This seems to be applicable for intrahepatic recurrence but also for isolated distant seedings. Yoh et al. [28] reported a 3-year survival rate of $86.7 \%$ in a total of 15 repeated resections, with 8 for metastatic disease, mainly in the lung [28]. Certainly, further data with special regard to the site and number of metastases are necessary to better evaluate the potential role of surgery in this setting.

Recurrence of iCCA, in particular after extended resections, is often located close to critical vascular or hilar structures, making redo surgery technically challenging. Nevertheless, perioperative mortality rates of $1.4-3.5 \%$ are considerably lower than those after first resections, most likely due to the limited extent of the second hepatectomies [20, 22, 23, 25, 27].

In the German study nearly half of the patients developed repeated recurrence after redo hepatectomy. In about one fourth of the cases repeated reresection was possible, with survival rates comparable to those after the first and second resections [29]. This is similar to what we already know from repeated resection of colorectal liver metastases. It is of particular interest as it indicates a subgroup of recurrent iCCA that remains confined to the liver. In these cases, application of liver-directed therapies, and in particular of repeated resection, appears obvious. No doubt, patients who are candidates for repeated resection are a highly selected group with a favorable tumor biology. Whether different patterns of recurrence, i.e., repeated sole intrahepatic versus diffuse extrahepatic spread, reflect biological heterogeneity of iCCA requires further attention and investigation.

The gathered data clearly underscore the need for a structured follow-up after resection in order to identify patients who are candidates for a redo operation as early 
as possible. In our own experience up to $50 \%$ of patients with isolated intrahepatic metastases and, though rare, even a few patients with isolated extrahepatic metastases might be candidates for surgery. This accounts for about $25 \%$ of all patients with recurrence, which is consistent with reports from other centers [20]. Since there is a remarkable number of late and often isolated metastases, follow-up should not be limited to 2 years.

The available data on whom and when to operate on for recurrent iCCA are still limited. One important aspect is the disease-free period. About one fourth of patients develop recurrence within 6 months. In these patients resectability is lower and the results are worse [26]. Tsilimigras et al. [26] reported a median survival of 9.3 months and a 5 -year-survival of $8.9 \%$. This poor outcome is a marker for an unfavorable biology of iCCA; thus particular caution should be exercised when indicating surgery. The use of preoperative chemotherapy to better select patients seems to be rational. We do not consider iCCA to be recurrent within 3 months after the initial resection for repeated surgery. Similarly, others do not go for surgery immediately after diagnosis but observe potential candidates for at least 3 months with or without chemotherapy [21]. This criterion is based on personal experience rather than objective data but aims for biological selection in an attempt to avoid futile surgery in rapidly growing and highly aggressive tumors.

Despite recent achievements the vast majority of patients with recurrent iCCA do not qualify for repeated resection. In selected cases with isolated hepatic relapse, local ablation therapy either by microwave (MWA) or by radiofrequency (RFA) is an alternative to surgery $[1,12$, 13]. In a nonrandomized study Zhang et al. [13] achieved a similar survival after local ablation versus surgical resection in solitary recurrent iCCA smaller than $3 \mathrm{~cm}$ (Table 1). In larger tumors there was a nonsignificant tendency towards a better survival after resection. Less perioperative morbidity is one advantage of MWA/RFA, but in cases with bilioenteric anastomosis particular attention is necessary due to an increased risk of liver abscess formation and infectious complications [33]. To further evaluate the effectiveness of local ablative therapies, data from randomized trials are needed.

In recurrent iCCA confined to the liver but not amenable to surgery or ablation intra-arterial treatment is a rational treatment option, offering a median survival between 7.6 and 13.2 months [11, 22, 27]. Reported data are difficult to assess, and comparability is hardly given particularly as often several techniques (TACE, SIRT, and intra-arterial chemotherapy) are lumped together. In a recent paper from Shanghai a meticulous analysis of TACE for recurrent iCCA in 183 patients is reported [10]. The median overall survival was 26.9 months, with a 1-, 3 - and 5-year-survival of $64.8,37.8$, and $21.4 \%$. These fa- vorable results deserve closer consideration. First, the iCCA differ from those in most Western countries with regard to risk factors for cholangiocarcinoma. In the Chinese paper more than half of the patients had an underlying hepatitis B infection - which has been reported to be a favorable prognostic factor in some studies $[34,35]$. Second, there were only 43 major but 140 minor initial resections, which is an almost inverse ratio in relation to those in reports from Western countries. Finally, prognostic factors of iCCA were comparably favorable in the paper from Shanghai (vascular invasion in only $5 \%$, lymph node metastases in only $14 \%$, and moderate or well-differentiated tumors in $73 \%$, with median CA $19-9$ levels almost within the normal range). This information shall not at all diminish the admittedly promising Chinese data but should be considered when comparing it with results from other studies.

In summary, treatment options for recurrent iCCA have been steadily improved in the palliative as well as in the potentially curative setting. Repeated resection offers a chance not only for prolonged survival but even for cure in some cases. In up to $15-25 \%$ of patients with recurrent iCCA a surgical approach is possible. With ongoing progress in chemotherapy and in targeted therapy, response rates as well as repeated resectability might increase $[5,8$, $9,36]$. We have to await further data of neoadjuvant treatment to draw valid conclusions regarding resection rates in primary as well as recurrent cholangiocarcinoma.

\section{Distal Cholangiocarcinoma}

Relapse after potentially curative resection of dCCA seems to be a little less frequent than after resection of pCCA or iCCA. Specific data on time of occurrence and distribution of disease are scarce. In a paper from Song et al. [6] from Seoul a recurrence rate of 35\% after resections of 474 extrahepatic (perihilar excluded!) bile duct carcinomas is reported. Of note, this series includes 120 sole segmental bile duct resections for carcinomas of the extrahepatic/extrapancretic biliary tree. In these cases the recurrence rate was $27.5 \%$ compared to $37 \%$ after pancreatoduodenectomy for more distally located cholangiocarcinomas [6]. In a Japanese multicenter study $54.8 \%$ of tumors recurred within 5 years. Relapse was locoregional in about one fourth of patients, most often isolated. The liver was by far the most frequent metastatic site followed by seedings in the peritoneum, lymph nodes, lungs and abdominal wall [37].

Surgery is rarely an option in recurrent dCCA. In the series from Nagoya and Seoul, reresections were possible in 16.3 and $7.9 \%$ of the cases, respectively. Song et al. reported on 13 surgical procedures including resection of distant metastases in four and pancreatoduodenectomies 
for local recurrence after initial segmental bile duct resection in nine cases [6]. In the series from Nagoya there was resection of locoregional tumor in three and of distant relapse in 10 patients [7]. Specific survival data are not given (Table 1).

In summary, treatment of recurrent dCCA usually is palliative. In selected cases there might be a place for surgery in distant metastases as well as in locoregional recurrence, mainly after an initial resection with a limited extent.

\section{Conclusion}

Recurrence after resection of biliary tract cancer is frequent. So far, palliative chemotherapy or even best supportive care only has been the standard of treatment in most patients. In recent years, radiological approaches such as TACE, RFA, MWA, or SIRT have broadened the therapeutic options. In addition, encouraging results have been reported for redo operations. This is particularly true for iCCA, where in a substantial number of pa- tients repeated resection was shown to increase survival and even offer the potential for cure. Although the improved outcome might be attributed to a careful patient selection and a favorable tumor biology repeated surgical treatment should be attempted whenever possible. Hence, it is of the utmost importance to better understand the routes of metastases and the patterns of distribution to define factors qualifying for repeated resection. This necessitates a structured follow-up after resection. Whether different patterns of recurrence after surgical resection may reflect not only different pathological characteristics but also biological heterogeneity and unique behavior requires further attention and investigation.

\section{Conflict of Interest Statement}

The author declares no conflict of interests.

\section{Funding Sources}

There are no funding sources for this work.

\section{References}

1 Bridgewater J, Galle PR, Khan SA, Llovet JM, Park JW, Patel T, et al. Guidelines for the diagnosis and management of intrahepatic cholangiocarcinoma. J Hepatol. 2014 Jun; 60(6):1268-89.

2 Rizvi S, Khan SA, Hallemeier CL, Kelley RK, Gores GJ. Cholangiocarcinoma - evolving concepts and therapeutic strategies. Nat Rey Clin Oncol. 2018 Feb;15(2):95-111.

3 El-Diwany R, Pawlik TM, Ejaz A. Intrahepatic Cholangiocarcinoma. Surg Oncol Clin N Am. 2019 Oct;28(4):587-99.

4 Lang H, Sotiropoulos GC, Sgourakis G, Schmitz KJ, Paul A, Hilgard P, et al. Operations for intrahepatic cholangiocarcinoma: single-institution experience of 158 patients. J Am Coll Surg. 2009 Feb;208(2):218-28.

5 Primrose JN, Fox RP, Palmer DH, Malik HZ, Prasad R, Mirza D, et al.; BILCAP study group. Capecitabine compared with observation in resected biliary tract cancer (BILCAP): a randomised, controlled, multicentre, phase 3 study. Lancet Oncol. 2019 May;20(5):66373.

6 Song SC, Heo JS, Choi DW, Choi SH, Kim WS, Kim MJ. Survival benefits of surgical resection in recurrent cholangiocarcinoma. J Korean Surg Soc. 2011 Sep;81(3):187-94.

7 Takahashi Y, Ebata T, Yokoyama Y, Igami T, Sugawara G, Mizuno T, et al. Surgery for recurrent biliary tract cancer: a single center experience with 74 consecutive resections. Ann Surg. 2015 Jul;262(1):121-9.

8 Zhang W, Zhou H, Wang Y, Zhang Z, Cao G, Song $\mathrm{T}$, et al. Systemic treatment of advanced or recurrent biliary tract cancer. Biosci Trends. 2020 Nov; 14(5):328-41.
9 Abou-Alfa GK, Sahai V, Hollebecque A, Vaccaro G, Melisi D, Al-Rajabi R, et al. Pemigatinib for previously treated, locally advanced or metastatic cholangiocarcinoma: a multicentre, open-label, phase 2 study. Lancet Oncol. 2020 May;21(5):671-84.

10 Ge Y, Jeong S, Luo GJ, Ren YB, Zhang BH, Zhang YJ, et al. Transarterial chemoembolization versus percutaneous microwave coagulation therapy for recurrent unresectable intrahepatic cholangiocarcinoma: development of a prognostic nomogram. Hepatobiliary Pancreat Dis Int. 2020 Apr;19(2):138-46.

11 Hyder O, Marsh JW, Salem R, Petre EN, Kalva $S$, Liapi E, et al. Intra-arterial therapy for advanced intrahepatic cholangiocarcinoma: a multi-institutional analysis. Ann Surg Oncol. 2013 Nov;20(12):3779-86.

12 Kamphues C, Seehofer D, Eisele RM, Denecke T, Pratschke J, Neumann UP, et al. Recurrent intrahepatic cholangiocarcinoma: single-center experience using repeated hepatectomy and radiofrequency ablation. J Hepatobiliary Pancreat Sci. 2010 Jul;17(4):509-15.

13 Zhang SJ, Hu P, Wang N, Shen Q, Sun AX, Kuang $M$, et al. Thermal ablation versus repeated hepatic resection for recurrent intrahepatic cholangiocarcinoma. Ann Surg Oncol. 2013 Oct;20(11):3596-602.

14 Yamamoto M, Takasaki K, Otsubo T, Katsuragawa H, Katagiri S. Recurrence after surgical resection of intrahepatic cholangiocarcinoma. J Hepatobiliary Pancreat Surg. 2001; 8(2):154-7.
15 Sotiropoulos GC, Lang H, Broelsch CE. Surgical management of recurrent intrahepatic cholangiocellular carcinoma after liver resection. Surgery. 2005 Jun;137(6):669-70.

16 Ohtsuka M, Kimura F, Shimizu H, Yoshidome H, Kato A, Yoshitomi H, et al. Significance of repeated resection for recurrent intrahepatic cholangiocarcinoma. Hepatogastroenterology. 2009 Jan-Feb;56(89):1-5.

17 Ercolani G, Vetrone G, Grazi GL, Aramaki O, Cescon M, Ravaioli $\mathrm{M}$, et al. Intrahepatic cholangiocarcinoma: primary liver resection and aggressive multimodal treatment of recurrence significantly prolong survival. Ann Surg. 2010 Jul;252(1):107-14.

18 Saiura A, Yamamoto J, Kokudo N, Koga R, Seki M, et al. Intrahepatic cholangiocarcinoma: analysis of 44 consecutive resected cases including 5 cases with repeat resections. Am J Surg. 2010;252:107-14.

19 Sulpice L, Rayar M, Boucher E, Pracht M, Meunier B, Boudjema K. Treatment of recurrent intrahepatic cholangiocarcinoma. $\mathrm{Br} \mathrm{J}$ Surg. 2012 Dec;99(12):1711-7.

20 Hyder O, Hatzaras I, Sotiropoulos GC, Paul A, Alexandrescu S, Marques H, et al. Recurrence after operative management of intrahepatic cholangiocarcinoma. Surgery. 2013 Jun; 153(6):811-8.

21 Noji T, Tsuchikawa T, Mizota T, Okamura K, Nakamura T, Tamoto E, et al. Surgery for recurrent biliary carcinoma: results for 27 recurrent cases. World J Surg Oncol. 2015 Feb; 13(1):82. 
22 Spolverato G, Kim Y, Alexandrescu S, Marques HP, Lamelas J, Aldrighetti L, et al. Management and outcome of patients with recurrent intrahepatic cholangiocarcinoma following previous curative-intent surgical resection. Ann Surg Oncol. 2016 Jan;23(1): 235-43.

23 Si A, Li J, Xing X, Lei Z, Xia Y, Yan Z, et al. Effectiveness of repeat hepatic resection for patients with recurrent intrahepatic cholangiocarcinoma: factors associated with longterm outcomes. Surgery. 2017 Apr;161(4): 897-908.

24 Miyazaki Y, Kokudo T, Amikura K, Kageyama Y, Takahashi A, Ohkohchi N, et al. Survival of surgery for recurrent biliary tract cancer: a single-center experience and systematic review of literature. Jpn J Clin Oncol. 2017 Mar;47(3):206-12.

25 Bartsch F, Paschold M, Baumgart J, HoppeLotichius M, Heinrich S, Lang H. Surgical resection for recurrent intrahepatic cholangiocarcinoma. World J Surg. 2019 Apr;43(4): 1105-16.

26 Tsilimigras DI, Sahara K, Wu L, Moris D, Bagante F, Guglielmi A, et al. Very early recurrence after liver resection for intrahepatic cholangiocarcinoma: considering alternative treatment approaches. JAMA Surg. 2020 Sep; 155(9):823-31.
$27 \mathrm{Hu}$ LS, Zhang XF, Weiss M, Popescu I, Marques HP, Aldrighetti L, et al. Recurrence patterns and timing courses following curative-intent resection for intrahepatic cholangiocarcinoma. Ann Surg Oncol. 2019 Aug; 26(8):2549-57.

28 Yoh T, Hatano E, Seo S, Okuda Y, Fuji H, Ikeno $\mathrm{Y}$, et al. Survival of recurrent intrahepatic cholangiocarcinoma: the impact and selection of repeat surgery. World J Surg. 2018 Jun; 42(6):1848-56.

29 Bartsch F, Eberhard J, Rückert F, Schmelzle M, Lehwald-Tywuschik N, Fichtner-Feigl S, et al.; German ICC collaboration group. Repeated resection for recurrent intrahepatic cholangiocarcinoma: a retrospective German multicentre study. Liver Int. 2020. doi: 10.1111/liv.14682.

30 Jarnagin WR, Ruo L, Little SA, Klimstra D, D'Angelica M, DeMatteo RP, et al. Patterns of initial disease recurrence after resection of gallbladder carcinoma and hilar cholangiocarcinoma: implications for adjuvant therapeutic strategies. Cancer. 2003 Oct;98(8): 1689-700.

31 Groot Koerkamp B, Wiggers JK, Allen PJ, Besselink MG, Blumgart LH, Busch OR, et al. Recurrence rate and pattern of perihilar cholangiocarcinoma after curative intent resection. J Am Coll Surg. 2015 Dec;221(6):1041-9.
32 Komaya K, Ebata T, Yokoyama Y, Igami T, Sugawara G, Mizuno T, et al. Recurrence after curative-intent resection of perihilar cholangiocarcinoma: analysis of a large cohort with a close postoperative follow-up approach. Surgery. 2018 Apr;163(4):732-8.

33 Yu MA, Liang P, Yu XL, Cheng ZG, Han ZY, Liu FY, et al. Liver abscess as a complication of microwave ablation for liver metastatic cholangiocarcinoma after bilioenteric anastomosis. Int J Hyperthermia. 2011;27(5):503-9.

34 Zhang XF, Chakedis J, Bagante F, Beal EW, Lv $\mathrm{Y}$, Weiss M, et al. Implications of intrahepatic cholangiocarcinoma etiology on recurrence and prognosis after curative-intent resection: a multi-institutional study. World J Surg. 2018 Mar;42(3):849-57.

35 Wang Q, Li J, Lei Z, Wu D, Si A, Wang K, et al. Prognosis of intrahepatic cholangiocarcinoma with HBV infection is better than those with hepatolithiasis after R0 liver resection: a propensity score matching analysis. Ann Surg Oncol. 2017 Jun;24(6):1579-87.

36 Le Roy B, Gelli M, Pittau G, Allard MA, Pereira B, Serji B, et al. Neoadjuvant chemotherapy for initially unresectable intrahepatic cholangiocarcinoma. Br J Surg. 2018 Jun; 105(7):839-47.

37 Komaya K, Ebata T, Shirai K, Ohira S, Morofuji N, Akutagawa A, et al.; Nagoya Surgical Oncology Group. Recurrence after resection with curative intent for distal cholangiocarcinoma. Br J Surg. 2017 Mar;104(4):426-33. 